DOI: http://dx.doi.org/10.33846/hn50406

http://heanoti.com/index.php/hn

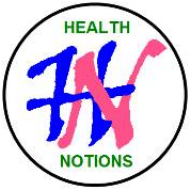

RESEARCH ARTICLE

URL of this article: http://heanoti.com/index.php/hn/article/view/hn50406

\title{
A Case Report of Placenta Percreta
}

\author{
Widiana Ferriastuti $^{1(\mathrm{CA})}$, Dwi P. R. Tampubolon ${ }^{2}$, Qonita ${ }^{3}$ \\ ${ }^{1(\mathrm{CA})}$ Department of Radiology, Faculty of Medicine, Universitas Airlangga, Indonesia; \\ widiana_ferriastuti@yahoo.com (Corresponding Author) \\ ${ }^{2}$ Research Asistant in Department of Radiology, Faculty of Medicine, Universitas Airlangga, Indonesia; \\ dwi.putri.rahayu-2017@ fk.unair.ac.id \\ ${ }^{3}$ Resident in Department of Radiology, Faculty of Medicine, Universitas Airlangga, Indonesia; \\ mrs.adisolech@gmail.com
}

\begin{abstract}
There has been an increased incidence of placenta accreta in recent decades, which is associated with an increase in cesarean delivery. A woman aged 39 years GIVP1111 at 8 months of gestation was a breech location with antepartum bleeding et. causa placenta previa totalis suspected percreta bladder infiltration and hematuria. The last abdominal ultrasound showed no visible clot retention and mild right-sided hydronephrosis (possibly a physiological condition). Due to doubts regarding the suspicion of placental invasion of the bladder, an MRI examination of the abdomen was performed. A network was irregular in shape and can not be oriented either right or left, some of which have been split. Attached to the placenta. It was not clear that the cervix and bladder were visible, the total weight was 500 grams, the size was $15 \times 13 \times 5 \mathrm{~cm}$. Based on both macroscopic and microscopic histopathological examinations, it could be concluded that the uterus, adnexa, surgery: placenta percreta, adenomyosis uteri.
\end{abstract}

Keywords: placenta percreta; uterus; antepartum bleeding

\section{INTRODUCTION}

The term lethal placental attachment represents abnormal implantation of the placenta in the uterine wall and is used to describe placenta accreta, increta, and percreta. Placenta accreta is a placenta in which the placental villi adhere directly to the myometrium, there is no invasion of the muscles; placenta accreta is the placenta where the placental villi invade into the myometrium; and placenta percreta is the placenta where the placental villi invade the entire thickness of the myometrium and enter the serosa. The depth of invasion is important clinically because of the necessity and the type of intervention based on it. Placenta accreta can be divided into: placenta accreta totalis, partial placenta accreta, placenta accreta fokalis based on the amount of placental tissue involved in attachment to the myometrium ${ }^{(1)}$.

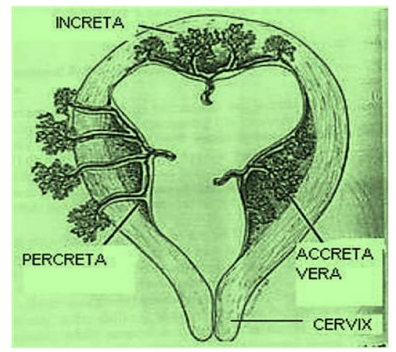

Figure 1. Placenta accreta classification

There has been an increasing incidence of placenta accreta in recent decades, which is associated with an increase in cesarean delivery ${ }^{(1-3)}$. The overall incidence of placenta accreta is approximately 3 cases per 1000 births, of which $75 \%$ is placenta accreta; $18 \%$ were placenta increta; and $7 \%$ were placenta percreta ${ }^{(1,3)}$. Cesarean delivery and placenta previa are the most important risk factors for placenta accreta ${ }^{(1,4)}$. Other risk factors include advanced maternal age, multiparity, history of previous uterine surgery, previous history of uterine radiation, endometrial ablation, Asherman's syndrome, leiomyoma uteri, uterine anomalies, hypertension in pregnancy and smoking ${ }^{(1)}$. In other literature it is suggested that dilation and curettation and myomectomy is a minor risk factor ${ }^{(4)}$. 
There is no clarity about placenta accreta's pathogenesis, in any case, a few hypotheses have been proposed. Irregular vascularization coming about from postoperative recurrent forms went with by auxiliary neighbourhood hypoxia driven to diminished viability of decidualization and the foremost unmistakeable thropoblastic attack, the hypothesis as of now most bolstred, clarifying the pathogenesis of placenta accreta at this stage ${ }^{(1)}$.

Most patients with placenta accreta are asymptomatic. Indications related with placenta accreta incorporate cramping and vaginal dying. These side effects have much to do with placenta previa, which is the most grounded hazard figure for placenta accreta. In spite of the fact that uncommon, the clinical potential for fiasco is intense stomach torment and hypotension from hypovolemic stun due to secondary uterine burst of placenta percreta. This will happen within the pregnancy prepare from the primary trimester to last term pregnancy without any signs of labour. Placenta accreta can cause many complications, including local organ damage, postoperative bleeding, amniotic fluid embolism, consumptive coagulopathy, transfusion-related complications, acute respiratory distress syndrome, postoperative thromboembolism, infectious morbidity, multi-organ failure, and maternal death. The invasion of the bladder by the placenta is a potentially lifethreatening obstetric complication, although rare, characterized by both abdominal pain and hematuria. Exact pre-birth conclusion is exceptionally vital to play down these dangers ${ }^{(1)}$.

The pathological sample received by hysterectomy will help to diagnose the placenta accreta. The conclusive determination is based on the appearance of the chorionic villi implanted within the myometrium without getting the decidual layer between them ${ }^{(1)}$.

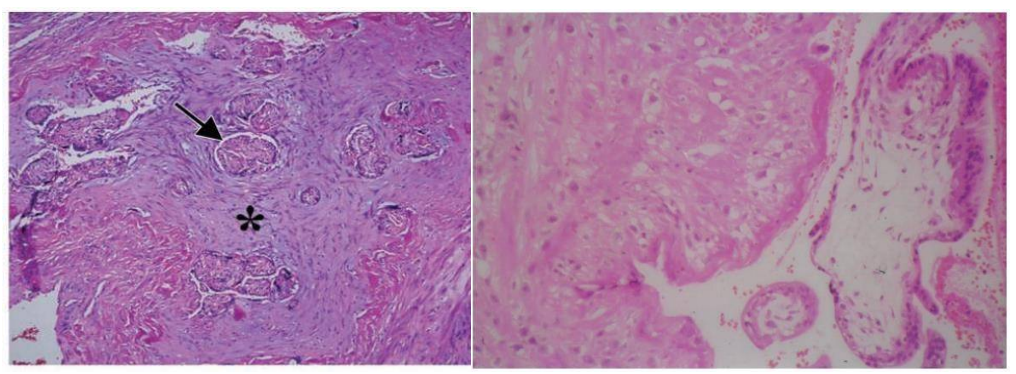

Figure 2. (a) Placenta acreta. Photomicrograph showing the chorionic villi (arrow) completely surrounded by a reactive myometrium $(*)$, consistent with placenta accreta. (b) Placenta perkreta. There are placental villi in the outer layer of the myometrium.

The preoperative diagnosis of placenta accreta can be directed by sonography and MRI . In common, grayscale sonography is a fabulous apparatus for the pre-birth determination of placenta accreta in ladies with chance components for the improvement of the clutter. The sensitivity is reported to be between $77 \%$ and $87 \%$ with a specificity of $96 \%$ to $98 \%$, PPV (positive predictive value) $65 \%$ to $93 \%$ and NPV (negative predictive value) $98 \%$ (2)
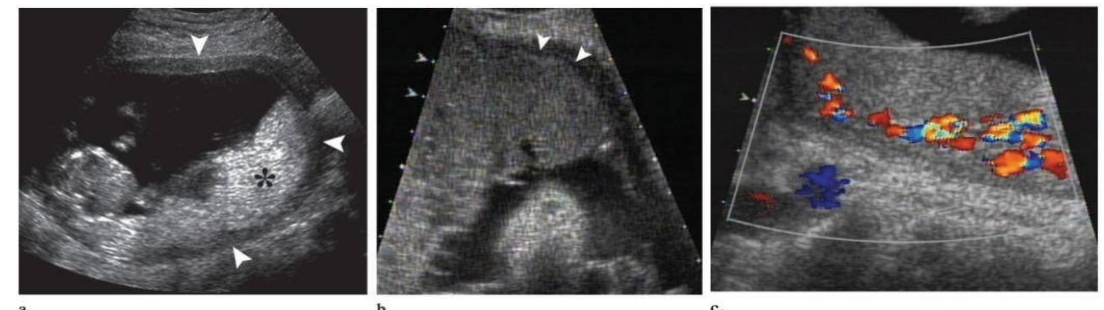

Figure 3.(a) Transverse sonography shows a hyperechoic $\left(^{*}\right)$ placenta surrounded by a hypoechoic myometrium. (b) Sagittal sonography shows a thin hypoechoic line (arrowhead) on the inside of the myometrium depicting subplacental clear space. (c) Sagittal sonography shows a normal pattern of subplacental blood flow parallel to the myometrium
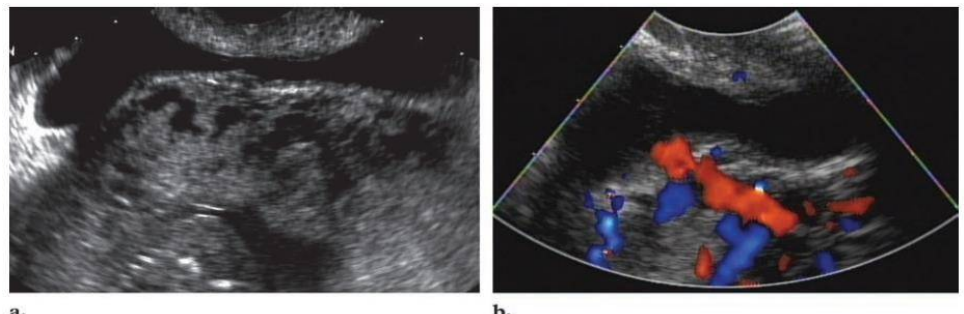

Figure 4. Lacunae placenta on placenta accreta. (a) Transvaginal transvaginal sonography shows multiple tortuous hypoechoic structures in the placenta. (b) Transverse transabdominal Doppler sonography helps confirm that the hypoechoic cleft is a blood vessel and is a placental lacuna 

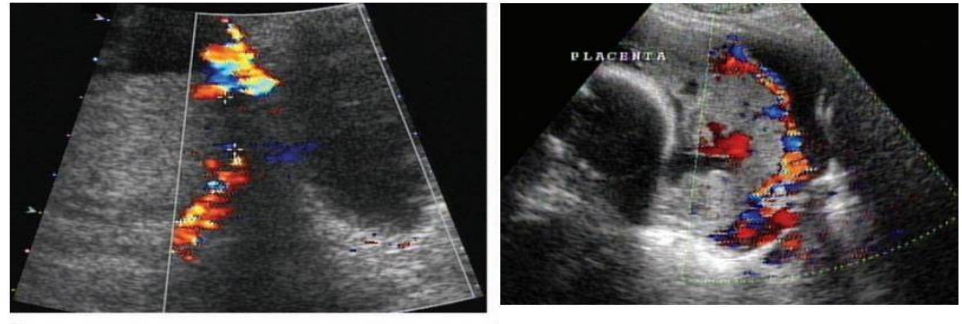

Figure 5. Placenta accreta. (a) Transabdominal sagittal sonography reveals a gap in myometrial blood flow in the lower uterine segment. (b) Doppler sonography sagittal transvaginal shows an increase vascularisation around the uterus with an overview placental lacunae
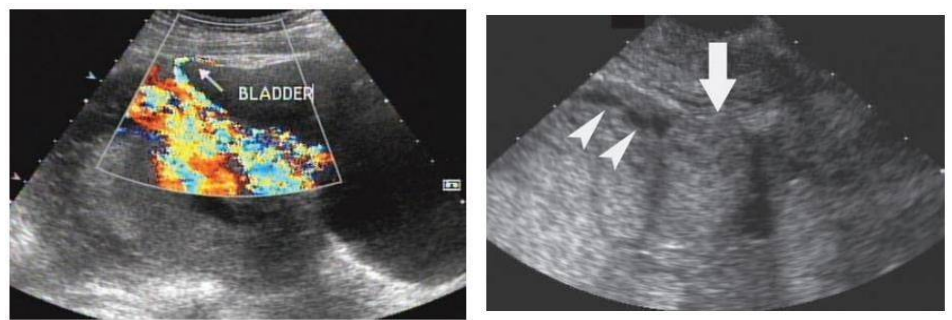

Figure 6. Placenta accreta. (a) On sagittal transabdominal Doppler sonography, extensive vascularization is seen along the anterior side of the lower uterine segment and appears to extend upwards and around the bladder. (b)

On sonography transvaginal sagittal, placenta seen across subplacental hypoechoic area (arrowheads) and appears protruding into the myometrium (arrow)
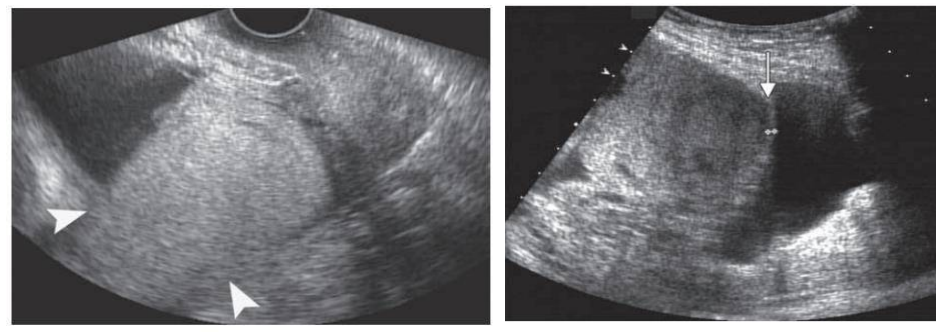

Figure 7. Placena accreta. (a) Transabdominal sagittal sonography shows the placenta extending posteriorly without a normal myometrium (arrowheads). There was also a loss of the retroplacental clear space image. (b) On sonography sagittal transabdomen, the placenta appears to be expanded to the serous surface of the bladder without involvement myometrium (arrow)

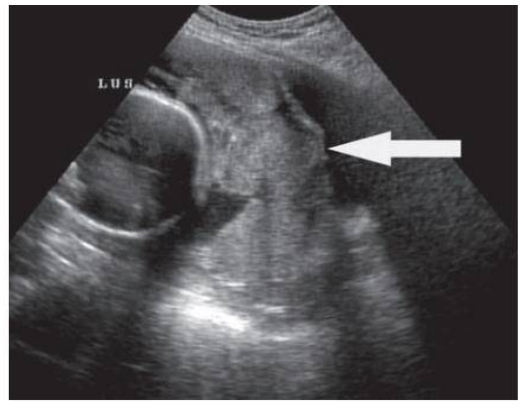

Figure 8. Placenta accreta. Sagittal transvaginal sonography shows placental lobulation extending to the edge of the bladder without myometrial involvement (arrow).

An important thing to highlight that when MRI is utilized in conjunction with sonography for the appraisal of patients analyzed with placenta accreta, it is once in a while supportive in changing surgical administration. In one think about, when MRI decreased the sonographic determination, in each case, the quiet was still subjected to a hysterectomy. The conclusion of placenta accreta from MRI can be either a wrong positive or a wrong negative ${ }^{(1)}$. 

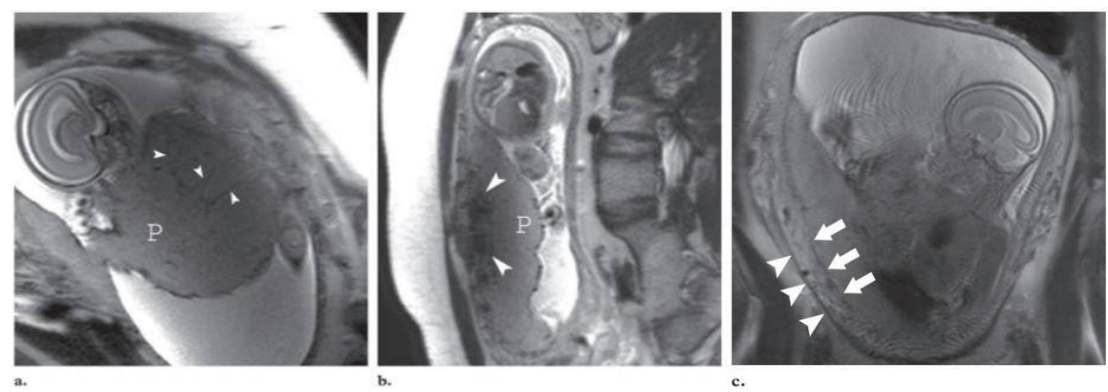

Figure 9. Image of normal placenta and myometrium on MRI. (a) MRI T2-weighted half-Fourier RARE image shows a homogeneous placenta $(\mathrm{P})$ with a thin linear area with decreased signal intensity in the normal pattern (arrowheads) representing normal placental septa. (b) T2-weighted half-Fourier RARE MRI image through the uterus shows a

homogeneous placenta $(\mathrm{P})$ with a tortous hypointense area below the placenta (arrowheads) depicting normal subplacental vascularization. (c) T2-weighted MRI image shows three normal layers of the myometrium, the outer and hypointense layers surrounding the more hyperintense middle layer containing blood vessels

Some of the placenta accreta features that can be found on MRI include: placenta previa, protrusion of the uterus, heterogeneous signal intensity in the placenta, intrapacental dark lines on T2-WI, focal interruption of the myometrial wall, bladder tension, direct picture of invasion of the pelvic structures by placental tissue.
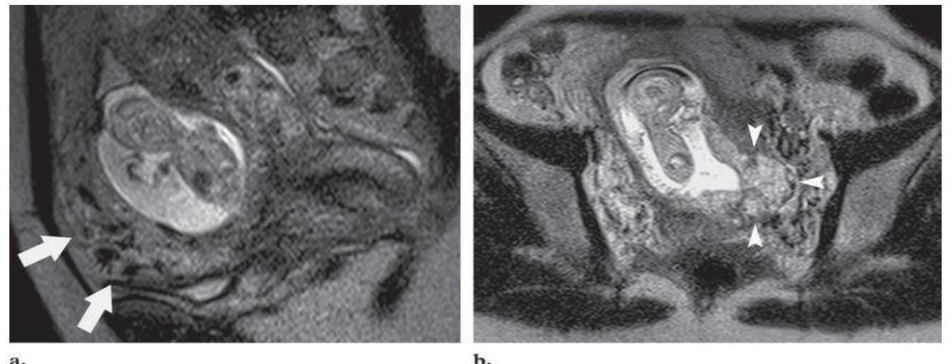

Figure 10. Placenta percreta. (a) MRI image of T2-weighted half-Fourier RARE showing protrusion of the anterior uterus (arrow) with widening of the lower uterine segment. (b) MRI T2-weighted half-Fourier RARE image shows a placenta with a higher signal intensity extending through the serous surface along the left and lateral posterior edges with parametrial invasion (arrowheads). There is also prominent vascularity (representing a number of tortuous void signals) around uterus

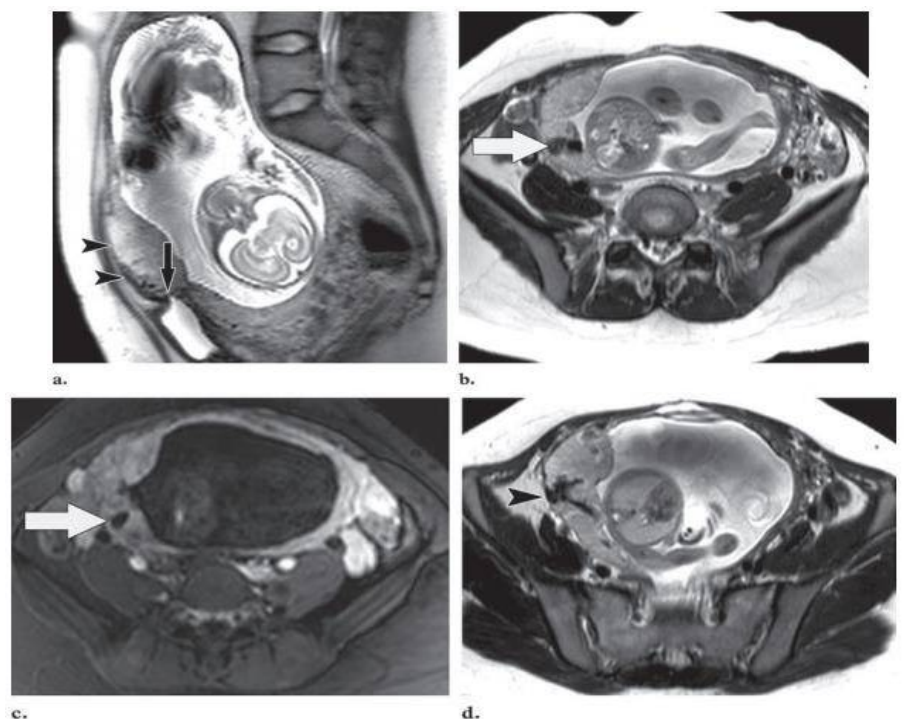

Figure 11. Placenta percreta. (a) MRI T2-weighted half-Fourier RARE image showing protrusion of the uterus (arrowheads). The "tenting" of the jar is also seen along its superior edge (arrow). (b, c) T2-weighted axial MRI image. (b) and T1-weighted contrast (c) shows areas of low signal intensity (arrows) indicating bleeding in the placenta (d) Axial T2-weighted half-Fourier RARE MRI image shows a placental line with low signal intensity extending from the myometrium-placenta surface (arrowheads) 


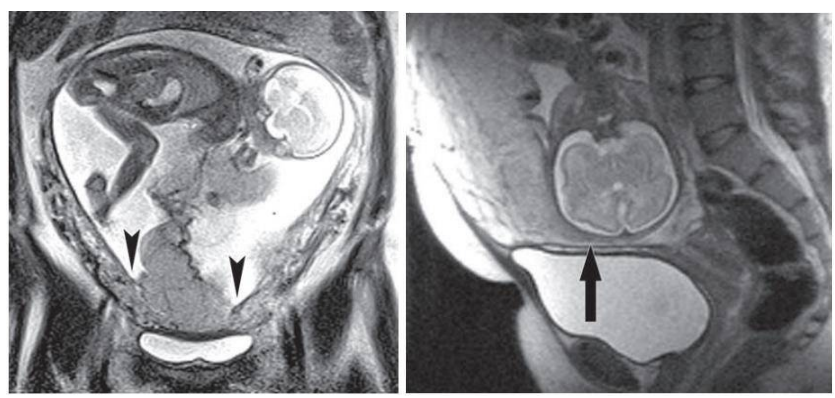

Figure 12. Placenta accreta. (a) Axial T2-weighted half-Fourier RARE MRI image shows a placental line with low signal intensity extending from the myometrium-placenta surface (arrowheads). (b) MRI shows a thin line of tissue which is slightly more hyperintense than the placenta. This finding is consistent with myometrial thinning in the lower uterine segment, which can be seen in normal pregnancy. Homogeneity of the placenta does not lead to placenta accreta

In addition, separated from 2-dimensional sonography and MRI, 3-dimensional sonography with control Doppler picture has been utilized to survey placental grips. Assesment of placental vascular design with 3dimensional control Doppler can offer assistance seperate placenta accreta from placenta percreta. Be that as it may, advance ponders are required for the broad utilize of 3-dimensional Doppler control ${ }^{(1)}$.

In general, the preferred course of action for patients with suspected placenta accreta is a planned preterm hysterectomy, with the placenta being left in situ because removal of the placenta can cause significant bleeding. In cases where a hysterectomy was not performed, additional therapy was performed, including hypogastric artery ligation, arterial embolization, methotrexate therapy, blood transfusions, antibiotics or curettage ${ }^{(2)}$.

\section{METHODS}

This study was a case report on a patient with a placenta percreta which was handled at the Dr. Soetomo hospital. The case was described with several difficulties likewise perpetual vaginal bleeding, severe abdominal pain and abnormal invasion of the bladder by the placenta, in which this case was handled by the collaboration with the obgyn and urologist.

\section{In Sumenep Hospital}

\section{RESULTS}

A higly risk pregnant patient with complaints of persistent bleeding during 1 week, followed by a severe abdominal pain the day before she came to the hospital. The patient had abortion and were curreted 19 years ago; moreover, she had an IUFD history 16 years before. An ultrasound examination of the abdomen was done with the results were the placenta located in the uterine body, not covering the birth canal, no visible retroplacental hypoechoic lesions, suspecting a blood clot in the bladder which became the referal criteria to the advance hospital.

\section{In Dr. Soetomo Hospital}

Furthermore, this woman was diagnosed regarding the difficulty in breech location, antepartum bleeding et.causa placenta previa totalis suspicious percreta, bladder infiltration, hematuria older than 35 years with an estimated fetal weight of 1000 grams. The incidence of placenta percreta is risen from 2015 to 2018, start with 7, 27, 60 and 71 cases respectively at Dr. Soetomo Hospital.

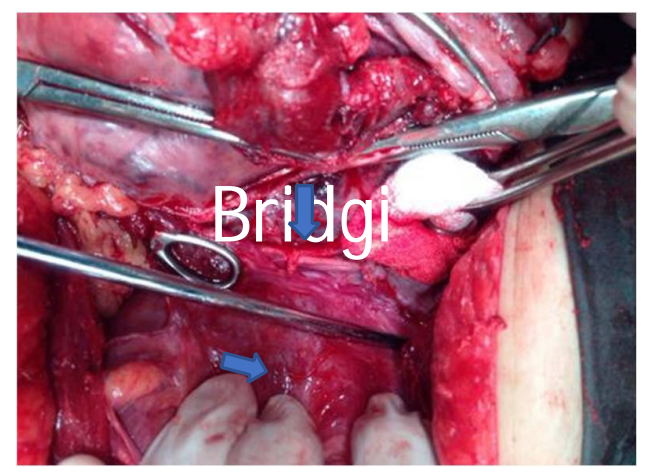

Figure 13. The post operation condition reported by the urologist after the Supervagibal Histerectomy (SVH) 
Active vaginal bleeding occurred suddenly after the patient returned from the MRI examination, the patient was in a pre-shock state (BP 110/80 mmHg, pulse 104 beats / minute, $\mathrm{Hb} 8.0 \mathrm{gr} / \mathrm{dl}$ ). At that time, emergency hysterotomy surgery with urology was planned up to the possibility of hysterectomy with all the risks until the bladder rupture. Urological operation report: uterine remains attached to the posterior wall of the bladder with a thin wall, evacuation of blood clots in the bladder (spooling through the urinary catheter until the urine is clear, urine mixed with pus \pm 1 liter, bladder is maintained (conservative).

On the 6th day after surgery, the problem arose, namely surgical wound infection (surgical wounds were treated 2 times / day with sugar, antibiotics, albumin transfusion (Alb 2.5) was also given to improve the patient's condition) Urine production as long as the treatment was sufficient (clear) and the patient had never had a fever (Hb $12.4 \mathrm{~g} / \mathrm{dl}, 10,700$ leukocytes), the infection of the surgical wound was getting deeper and wider. Two weeks after surgery (day 15), urine mixed with pus mixed with necrotic tissue and $+300 \mathrm{ml}$ from the urinary catheter, while $+1100 \mathrm{ml}$ from the abdominal drain, suspected an enterocutaneous fistula or abdominal burst and a relaparotomy was planned.

Relaparotomy was performed on the 20th day postoperatively along with urologic and digestive surgery, the operation was obtained: great attachment of the omentum to the sigmoid colon and vaginal stomp, posterior bladder rupture $+6 \mathrm{~cm}$ transverse, $+4 \mathrm{~cm}$ from the bladder neck, severe adhesions at the wound edge posterior bladder) accompanied by a very fragile bladder tissue, an open cystostomy and bladder repair were performed, the fascia was sutured again and placed a stent hecting. Ultrasound Urological evaluation: suspicious of remaining placental tissue adhering to the postero-superior wall of the bladder (bladder infiltration?), Suspect loculated ascites. The cystostomy catheter was maintained and planned to be removed after cystographic evaluation if there was no bladder leakage.

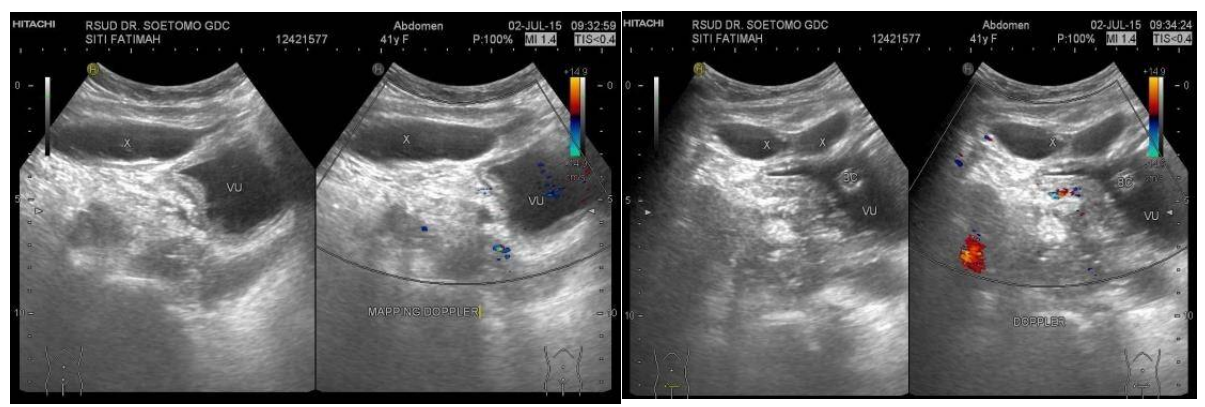

Figure 14. The evaluation of bladder post relaparatomy

Cystostomy catheter removed 28th day post relaparotomy. There were no complaints when urinating and the surgical wound began to dry. The patient was treated for 72 days, and then a control in the obstetrician for treatment of the surgical wound that was nearly healed. There were no other complaints while the patient was at home, while the baby died after being admitted to the NICU for 30 days.

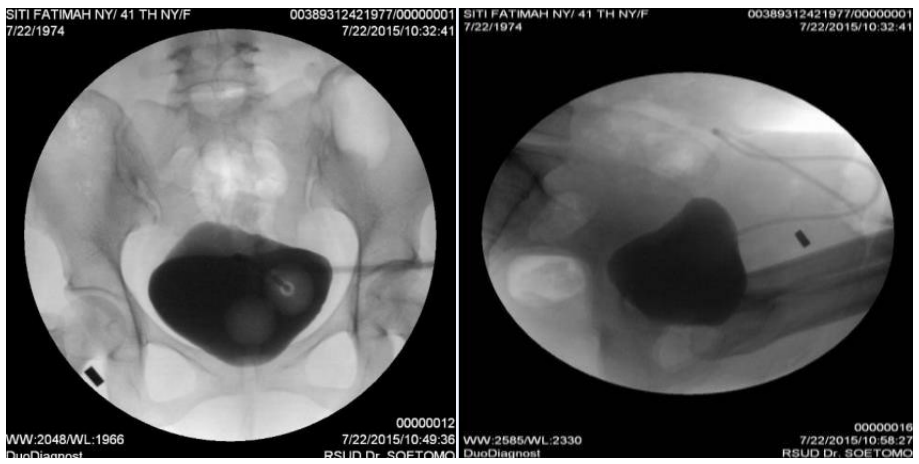

Figure 15. Cystostomy. Intact bladder, no visible extravasation of contrast. Bladder deformity and irregularities in the posterior wall of the bladder suggest postoperative brushing

\section{DISCUSSION}

Although rare, hypotension and hypovolemic shock are also seen in these patients due to profuse vaginal bleeding due to bladder / uterine rupture. Thus, in this patient it was decided to do surgery immediately to reduce maternal mortality even though the pregnancy was still preterm. The main risk factors for placenta accreta patients are a history of cesarean delivery and the presence of placenta previa ${ }^{(1,4)}$. Other risk factors

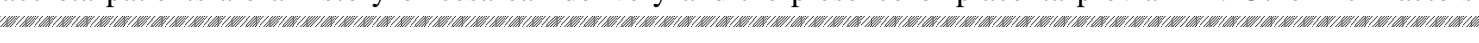
$\mathbf{1 4}$ | Publisher: Humanistic Network for Science and Technology 
include advanced maternal age, multiparity, history of previous uterine surgery, history of previous uterine radiation, endometrial ablation, Asherman's syndrome, leiomyoma uteri, uterine anomalies, hypertension in pregnancy and smoking as well as dilatation and curettage ${ }^{(1)}$. In this patient, several risk factors are found: the presence of placenta previa, advanced maternal age (39 years), and a history of dilation and curettage. The presence of adenomyosis in the above patients (from the results of postoperative PA) can also be a risk factor.

The presence of clinical suspicion of placenta percreta is supported by an image on abdominal ultrasound where placenta praevia and irregularity of the posterior wall of the bladder are obtained, accompanied by vascularization of the placenta to the posterior wall of the bladder.

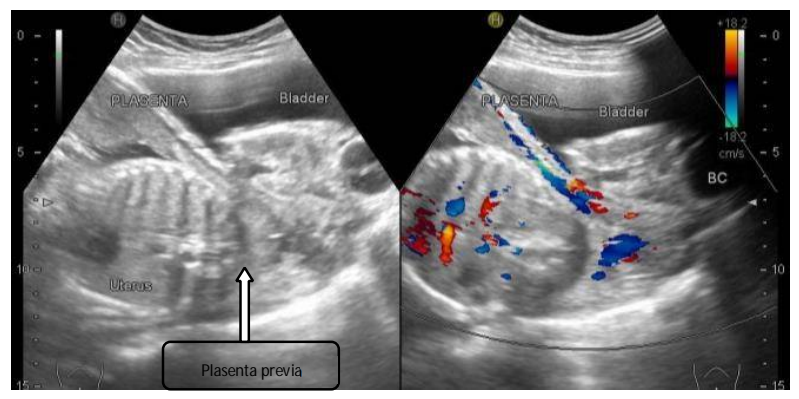

Figure 16. Abdomen imaging of placenta previa

This was confirmed by MRI examination where there was a bulging of the uterus and there was no normal fat-line from the right posterior wall of the bladder accompanied by multiple flow void images in the area which contributed to the infiltration of the placenta to the right posterior wall of the bladder. During the operation, there was a posterior wall of the bladder that was attached and difficult to remove by SBR and showed a lot of vascularization, according to the image on MRI.

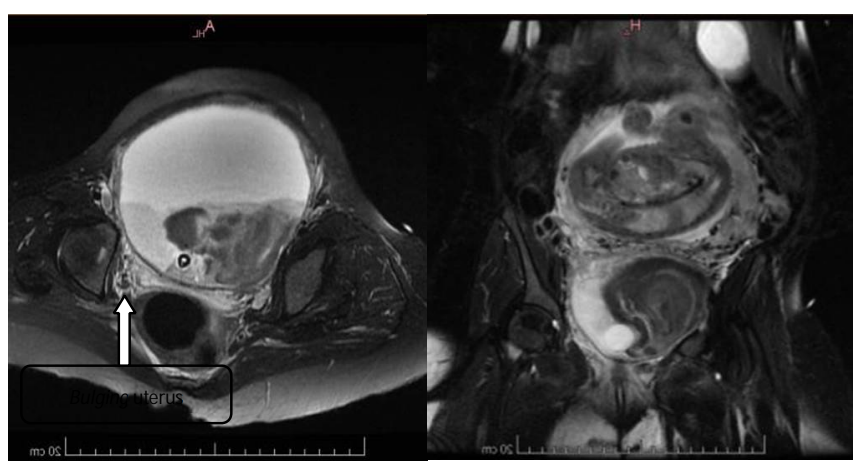

Figure 17. The presence of pus mixed with urine obtained in the bladder after surgery indicates an infectious process that may occur secondary to placenta accreta

\section{CONCLUSION}

Based on both macroscopic and microscopic histopathological examinations, it could be concluded that the uterus, adnexa, surgery: placenta percreta, adenomyosis uteri.

\section{REFERENCES}

1. Berkley EM, Abuhamad AZ. Prenatal Diagnosis of Placenta Accreta. Am Inst Ultrasound Med. 2013;32(April):1345-50.

2. Placenta Accreta. Am Coll Obstet Gynocologists. 2012;120(529):207-11.

3. Norris BL, Everaerts W, Posma E, Murphy DG, Mark P, Costello AJ, et al. The urologist 's role in multidisciplinary management of placenta percreta. BJU Int. 2016;117:961-5.

4. Varghese B, Singh N, George RAN, Gilvaz S. Magnetic resonance imaging of placenta accreta. Indian J Radiol Imaging. 2013;23(4). 